\title{
Behavior under Load of A Human Shoulder: Finite Element Simulation and Analysis
}

\author{
Manuel Islán Marcos ${ }^{1} \cdot$ Emilio Lechosa Urquijo ${ }^{1} \cdot$ Fernando Blaya Haro $^{1} \cdot$ Roberto D'Amato $^{1}$ (D) Enrique Soriano Heras ${ }^{2}$. \\ Juan Antonio Juanes ${ }^{3}$
}

Received: 8 January 2019 / Accepted: 14 March 2019 / Published online: 2 April 2019

(C) Springer Science+Business Media, LLC, part of Springer Nature 2019

\begin{abstract}
Most musculoskeletal injuries occur during the work routines in different areas, due to repetitive and sustained movements, they are often located in the shoulder. For workers in the building sector, the repetitive movements and displacements occur under load and unnatural postures of the shoulder joint. For this reason, this study aims to model in 3D the biological components which form the shoulder joint for the later finite element analysis. Three cases with different loads have been considered for this study. Due to a linear and isotropic joint approach it has been possible to evaluate the tensions in the main components of the shoulders: muscles, tendons and ligaments. The methodology used allowed obtaining an improved mesh of the shoulder joint to analyse real situations with finite element method analysis with applications in the field of sports medicine, work, etc. Furthermore, the simplification adopted for modelling the joint muscles, as 1D elements in the finite element model has made it possible to establish different positions of human joint without mesh again each of the studied positions. The results are consistent with the states of applied loads. In fact, the maximum stresses in bones are in the insertion areas of the ligaments. Due to the static positions of the joint under study, the muscles do not support high stresses. According to the stresses distribution, the maximum values are in the zones of tendons. From the result analysis, it is observed how the stresses distribution in the cartilage area maintains coherence with reality since the maximum stresses appear in the lower half of the cartilage. In this area in which the Humerus-scapula contact is greater, the compression tensions are greater.
\end{abstract}

Keywords Finite element analysis $\cdot$ Shoulder $\cdot$ Humerus $\cdot$ Muscles $\cdot$ Tendons $\cdot$ Ligaments

\section{Introduction}

Due to its high mobility, the shoulder is the Human joint that has the most injuries in its tissues [1]. The rotator cuff is formed by the tendons of the subscapular, supraspinatus, infraspinatus, and minor rotator cuff muscles that meet at the shoulder joint $[2,3]$. It is placed in the arch called coracoacromial, constituting the

This article is part of the Topical Collection on Education \& Training

Manuel Islán Marcos

manuel.islan.marcos@upm.es

Roberto D'Amato

r.damato@upm.es

Juan Antonio Juanes

jajm@usal.es

Emilio Lechosa Urquijo

emilio.lechosa@upm.es

Fernando Blaya Haro

fernando.blaya@upm.es
Enrique Soriano Heras

esoriano@ing.uc3m.es

1 Escuela Técnica Superior de Ingeniería y Diseño Industrial, Universidad Politécnica de Madrid, Ronda de Valencia, 3, 28012 Madrid, Spain

2 Dpto. de Ingeniería Mecánica, Universidad Carlos III de Madrid, Avda. de la Universidad, 30, 28911 Leganés, Madrid, Spain

3 Campus Miguel de Unamuno, Universidad de Salamanca, 37007 Salamanca, Spain 


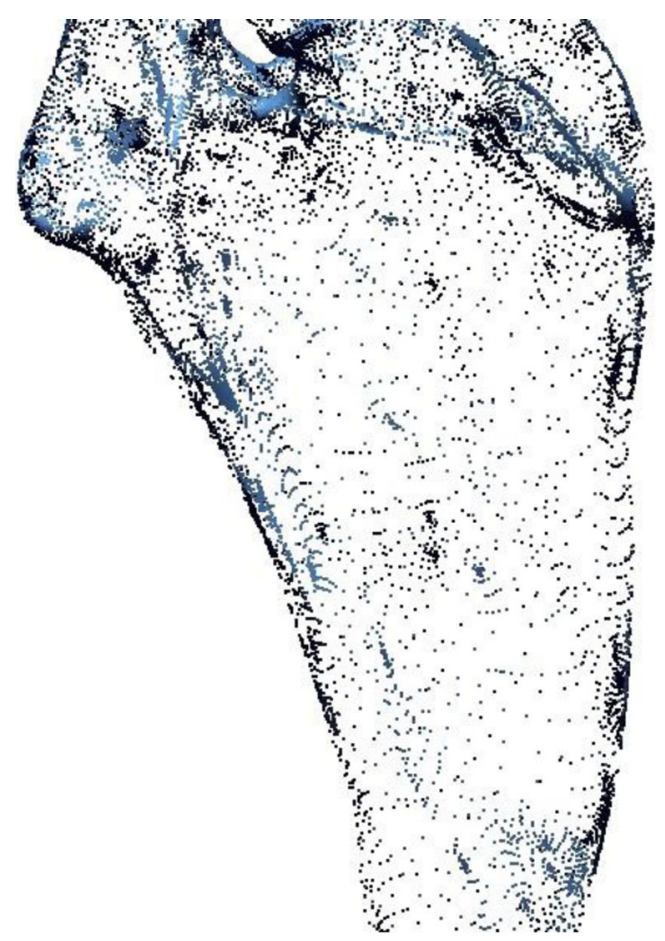

Fig. 1 Cloud of points obtained through a resonance

subacromial space with a serous pouch that lubricates the cuff. This pathology is characterized by progressive pain as a result of an accumulated overload or acute after an overexertion, which is located on the upper or lateral side of the shoulder and increases with movement, mainly with the elevation of the arm (ex. when

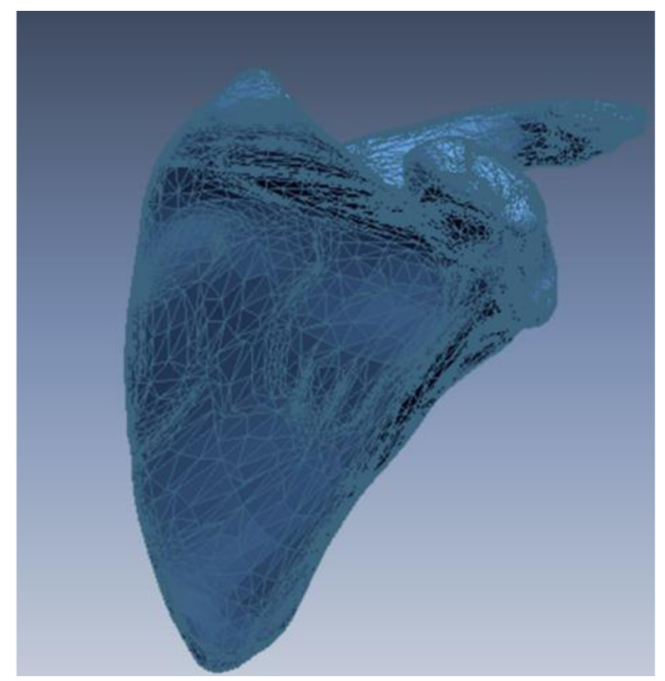

Fig. 2 Mesh corresponding to the scapula combining) and with internal rotation (ex. when putting on a jacket) [4]. The pain may be more acute at night when leaning on the affected side. The chronic injury can cause a rupture of the tendons of the cuff. Chronic tendinitis of the rotator cuff may be related to shoulder overload, joint instability and rotator cuff degeneration with age. It is relatively frequent, reaching up to $18 \%$ in workers in heavy manual tasks with great efforts on the shoulders, associated or not with repetitive movements and forced postures. $75 \%$ of people over 50 years have rotator cuff injuries and most of them are asymptomatic [5]. Many jobs in different trades in the construction sector involve important musculoskeletal efforts. This pathology is associated with work that involves stabilizing the shoulders to maintain or move the elbows in an elevated position by force, lifting and catching continuously, or working the arm in flexion or abduction. In the construction sector, this occurs in the assembly of metallic or prefabricated structures, also of heavy prefabricated elements that must be manipulated to unload. In order to understand the behaviour of human joints, in recent years, several authors have proposed scientific studies with finite element model [6] as well as the studies conducted to simulate the dynamic behaviour of human joints. For example, Ruggiero et al. in their study present a new mathematical model in order to understand the tribological performances of the human ankle joint with synovial fluid filtrated by articular cartilage in the case of Newtonian fluid and of no Newtonian fluid [7, 8]. D'Amato et al. in their study investigate the importance of the morphometric parameter of the human joint for the mathematical modelling of the human ankle joint [9].- Many authors, in recent years, have focused their studies on the description of the dynamic behavior of the shoulder joint. For example, in the 1998 Luo et al. present for the first time a simplified 2D-finite element model in order to described the stress environment in the supraspinatus tendon [10]. Wakabayashi et al. in the 2003 performed 2D-finite element model analysis in order to estimate the mechanical environment of the supraspinatus tendon. In the 2008 Seki et al. [11] presented the firs 3D-finite element model of rotator cuff tears in order to study the stress distribution in the supraspinatus tendon. Most of these studies have a substantial limitation. In fact, in these studies only the scapula and the bones of the Humerus were considered. This means that in presenting their results both in the $2 \mathrm{D}$ and $3 \mathrm{D}$ geometry, the structure of the muscles and other soft tissues have been neglected [12]. For this reason, in this study a finite 

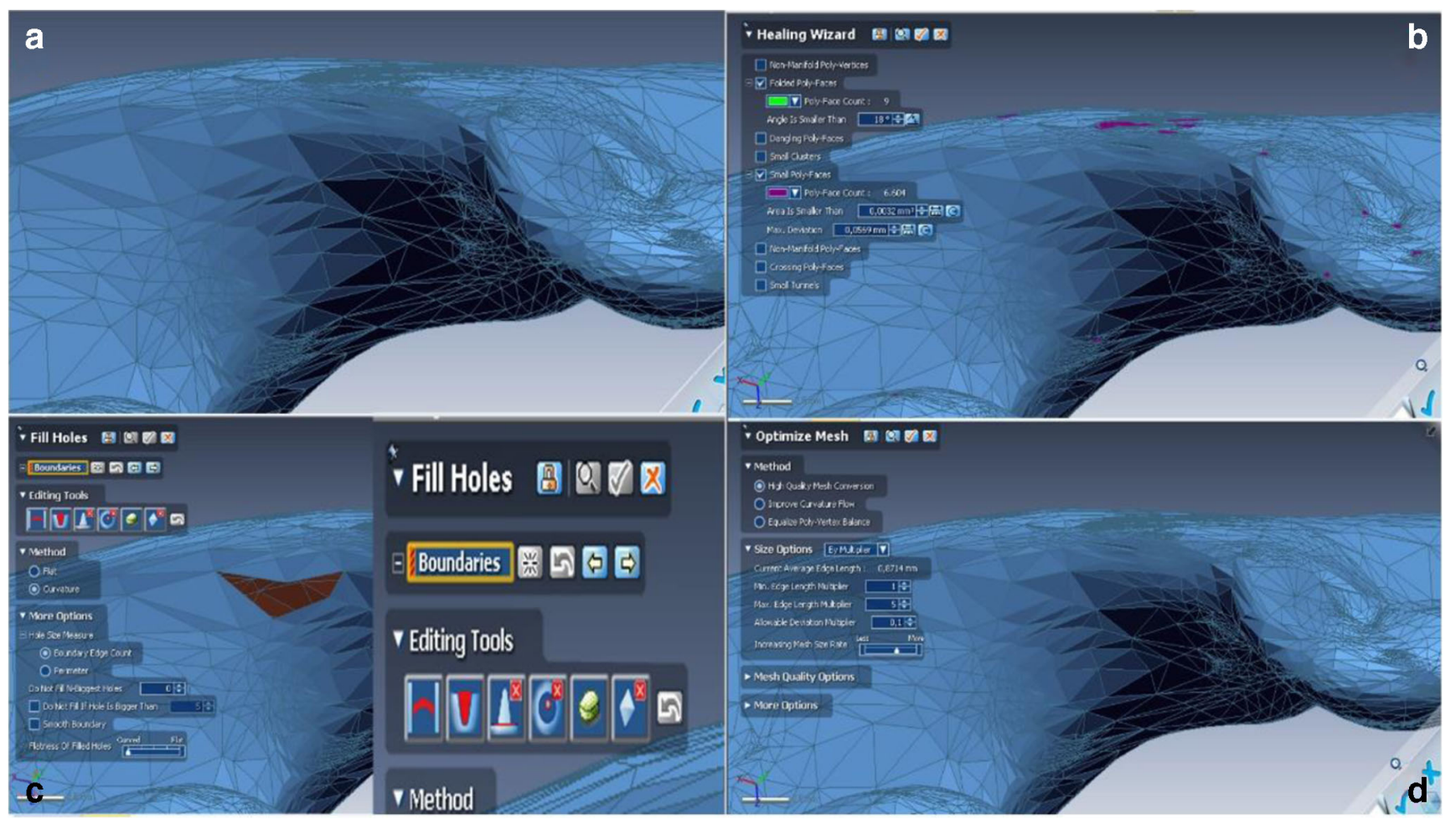

Fig. 3 Mesh and cloud of points operations

element model of the shoulder joint, glenohumeral, classified as a diarthrosis, of the genus enarthrosis or spheroid is presented. Based on the finite element model made for the case of a violinist [3] they are carried out modifications and improvements that allow us increasing the accuracy of the study and improving the predictive performance of it. This is due to include the ligaments, glenohumeral and coracohumeral, and the glenohumeral-capsule in the anatomical model. In the same way the meshing process is improved, by working

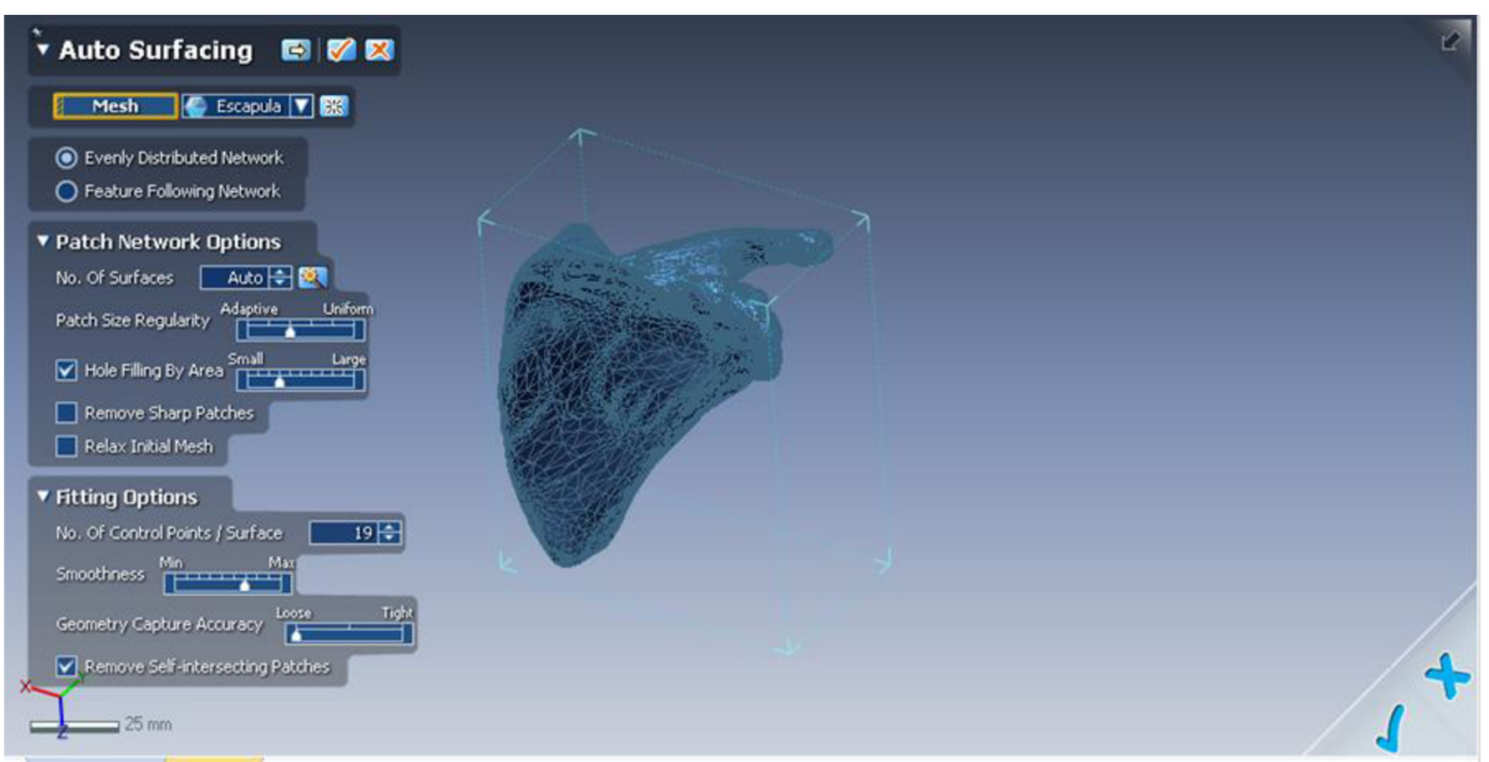

Fig. 4 Reconstruction of surfaces from the cloud of points 


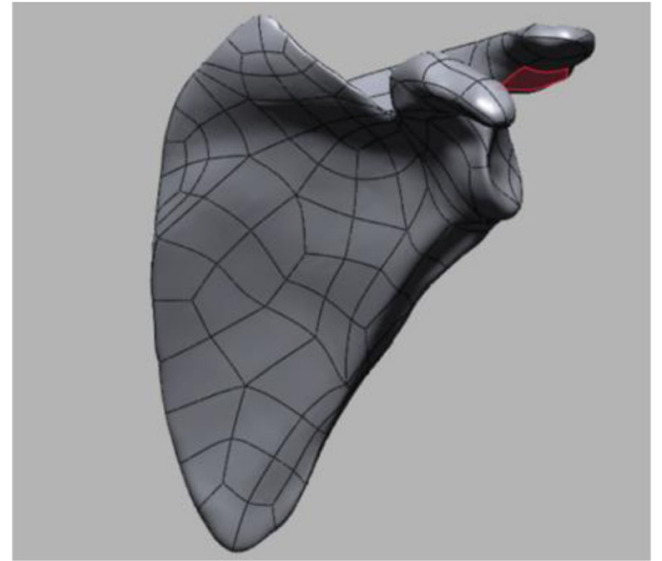

Fig. 5 Surface generated by Geomagic Design $\mathrm{X}$ associated with the scapula

on the characterization of the tendon-bone insertion, achieving a transfer of efforts between both more precise and in accordance with reality.

The study focuses on a unique position shoulder, flexion $53^{\circ}$. The scope of work aims the next aspects:

- Improving the mesh used in the finite element model.

- Improving the characterization of the contact tendon-bone insertion area. To do this, meshes between both elements have been made congruent, with nodes coinciding on the interface surface.

- Including the main ligaments involved in the joint, glenohumeral ligaments (lower, middle and upper) and coracohumeral ligament.

Fig. 6 Process to model tendons

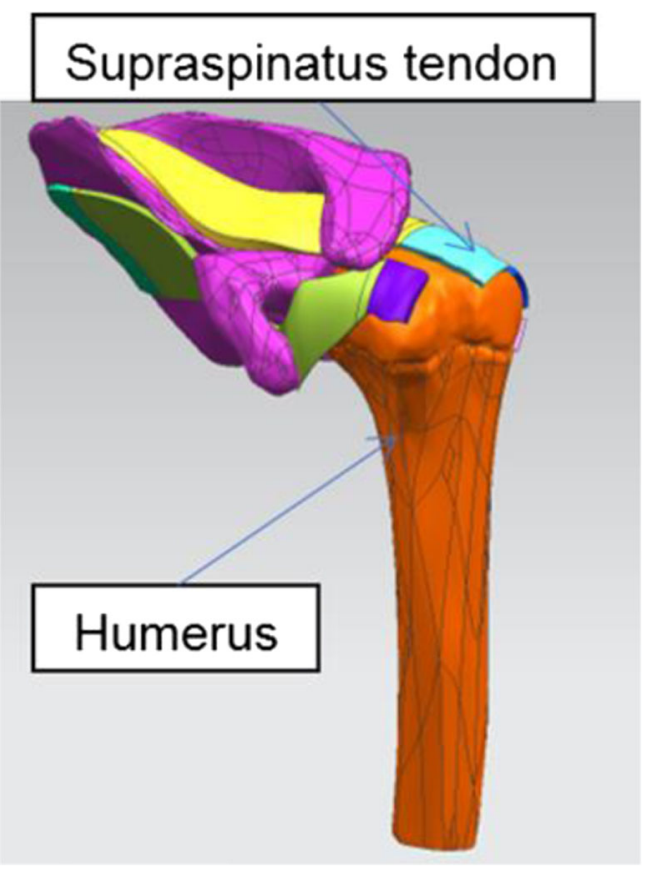

Fig. 7 Location of the supraspinatus tendon and the head of the humerus

- Obtaining a qualitative model of joint behavior.

\section{Methodology}

The process to generate a 3D model from a cloud of points is described in the following steps:

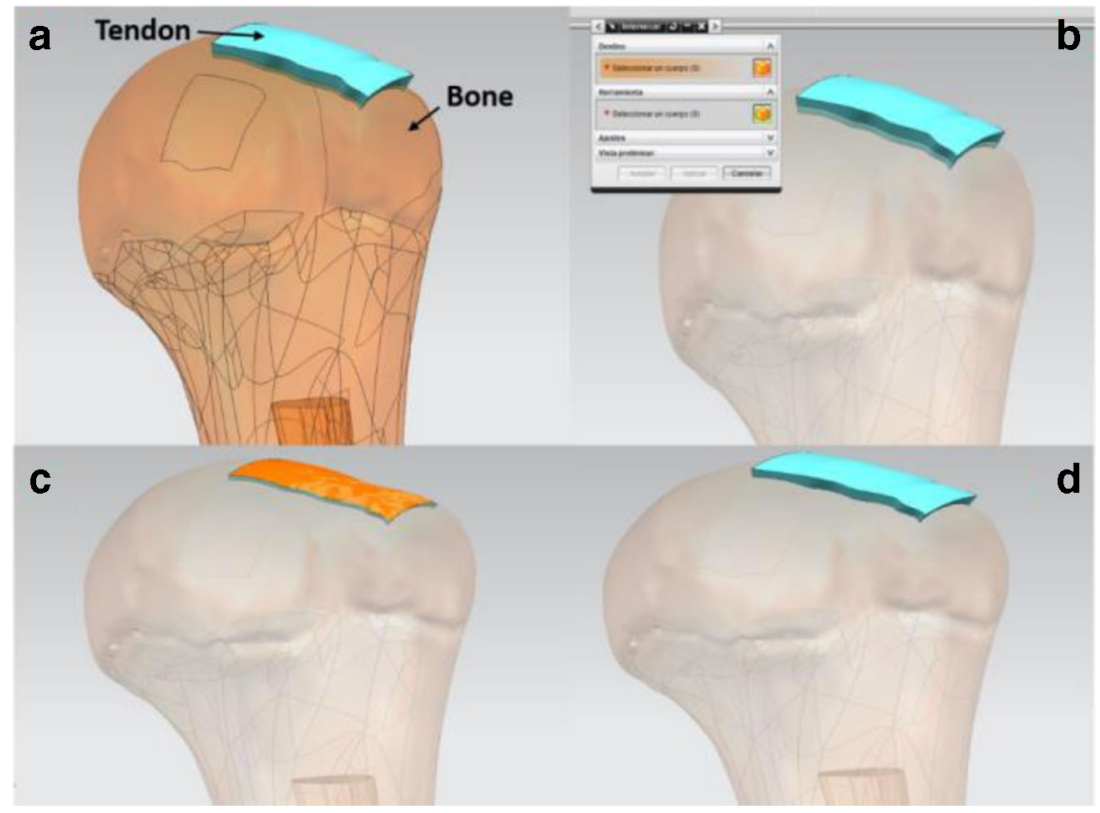




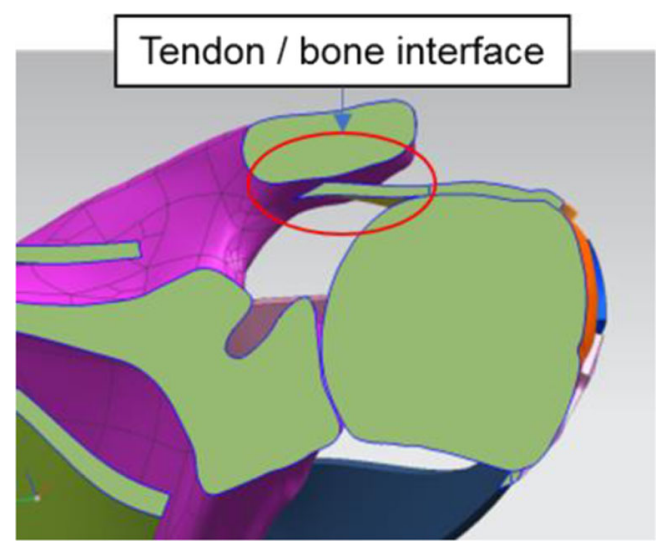

Fig. 8 Sectional view of the humerus + supraspinatus tendon

Starting from the cloud of points (Fig. 1) obtained through a resonance. A 3D Computer Aided Design (CAD) model of the elements involved in the scapula.

Using the software Geomagic Design X, it is possible to create a mesh supported in the obtained cloud of points (Fig. 2). The mesh is a triangulation of the surface of the object joining the points of the cloud.

Among the various operations that can be performed using the software Geomagic Desing X, all of them are aimed at improving the cloud of points and its associated mesh, are the following (Fig. 3):

- Repairing the mesh, by creating points in the cloud, based on the existing points in it (Fig. 3a).

- Filling holes in the mesh. The mesh is completed creating the triangles and points in the cloud that are missing to close the outer surface of it (Fig. 3b). The filling of holes can be done in different ways (Fig. 3c).

- Optimizing the mesh. It is possible to simplify the number of points in the cloud, eliminating both duplicated points and points which do not provide information, decreasing the size of the cloud (Fig. 3d).

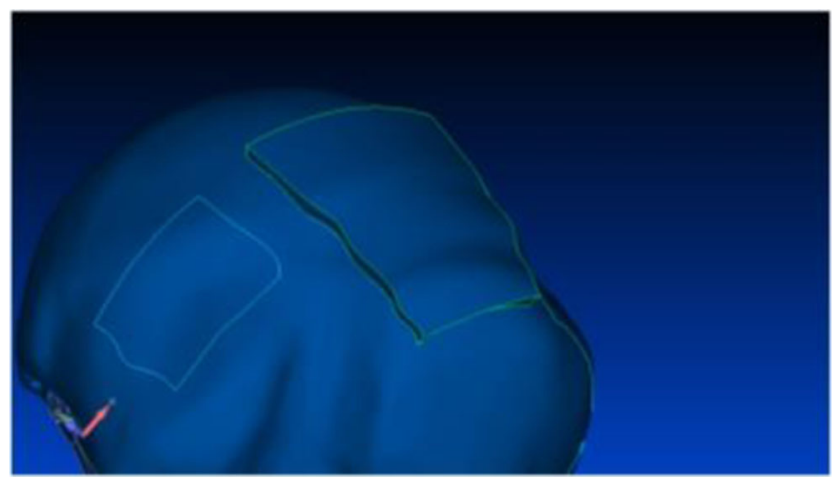

Fig. 9 View of the Humerus + supraspinatus tendon

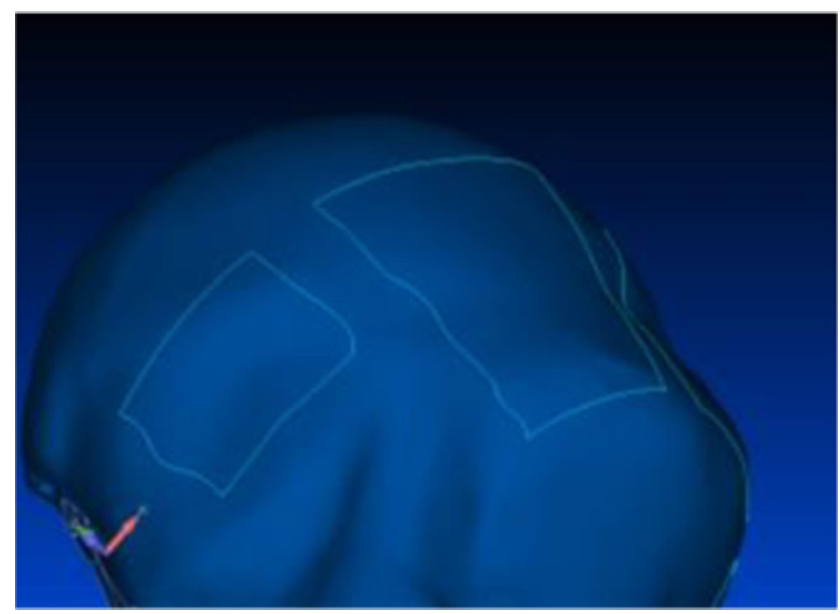

Fig. 10 Outline of the tendon projected on the Humerus

In addition to these operations, there are many other operations aimed at modifying the cloud of points such as symmetry operations, copy points, scaling of the piece, etc. These operations are also included in commercial Computer Aided Design (CAD) systems,

The most important operation that Geomagic Design $\mathrm{X}$ offers us, is the reconstruction of surfaces based on the cloud of points. The result of this operation is an entity that can be treated in any commercial CAD system. The importance of this operation lies in the possibility of using the reconstructed entity for other purposes such us modification of the geometry in a commercial CAD system, integration of the entity in a set of pieces, finite element analysis operations.

Table 1 Types of elements for each body that intervenes in the joint

\begin{tabular}{|c|c|c|c|}
\hline $\mathrm{N}^{\circ}$ & Element type & Parameters & Application \\
\hline 1 & $\begin{array}{r}\text { Tetrahedral } \\
10 \text { nodes }\end{array}$ & $\begin{array}{l}\text { Material, Young } \\
\text { modulus and } \\
\text { Poisson coef. }\end{array}$ & $\begin{array}{l}\text { Meshing bones, } \\
\text { muscles and tendons }\end{array}$ \\
\hline 2 & $\begin{array}{l}\text { Beam element } \\
2 \text { nodes }\end{array}$ & $\begin{array}{l}\text { Material, Young } \\
\text { modulus and } \\
\text { Poisson coef. and } \\
\text { transverse section }\end{array}$ & $\begin{array}{l}\text { Mesh of the } \\
\text { ligaments and the } \\
\text { glenohumeral } \\
\text { capsule. }\end{array}$ \\
\hline 3 & $\begin{array}{l}\text { Rigid element } \\
2 \text { nodes }\end{array}$ & none & $\begin{array}{l}\text { Simulation insertion } \\
\text { of the ligament in } \\
\text { the bone. }\end{array}$ \\
\hline 4 & $\begin{array}{l}\text { Gap element } \\
2 \text { nodes }\end{array}$ & $\begin{array}{c}\text { Gap and stiffness } \\
\text { of the contact. }\end{array}$ & $\begin{array}{l}\text { Simulation of the } \\
\text { cartilage contact } \\
\text { between the head } \\
\text { of the Humerus and } \\
\text { the scapula. }\end{array}$ \\
\hline
\end{tabular}




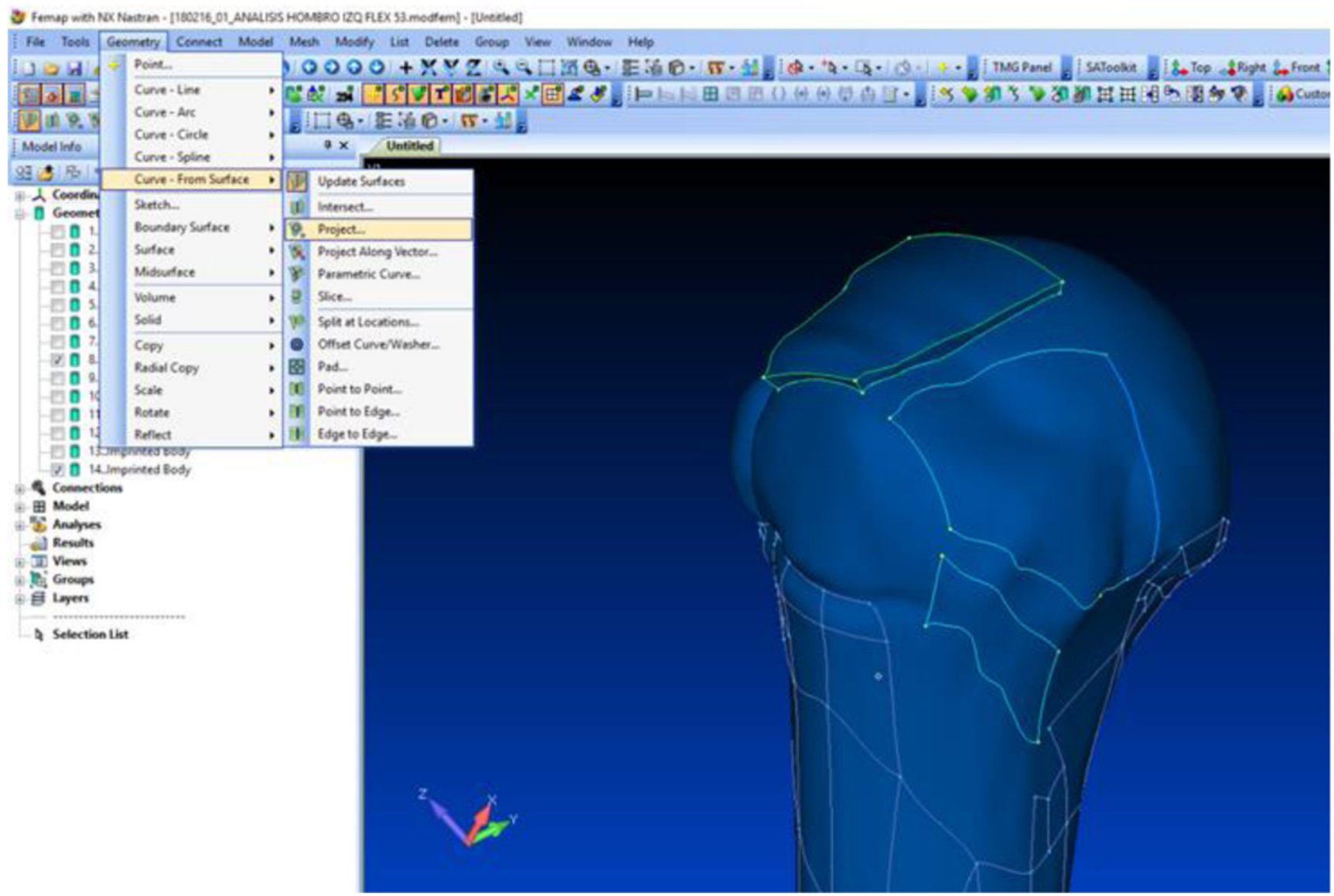

Fig. 11 Contour of the tendons in Siemens FEMAP

The reconstruction of the cloud of points can be done in two different ways:
- Based on a uniformly distributed network. Geomagic Design $\mathrm{X}$ is responsible for generating "patches" based
Fig. 12 Projection of the contour

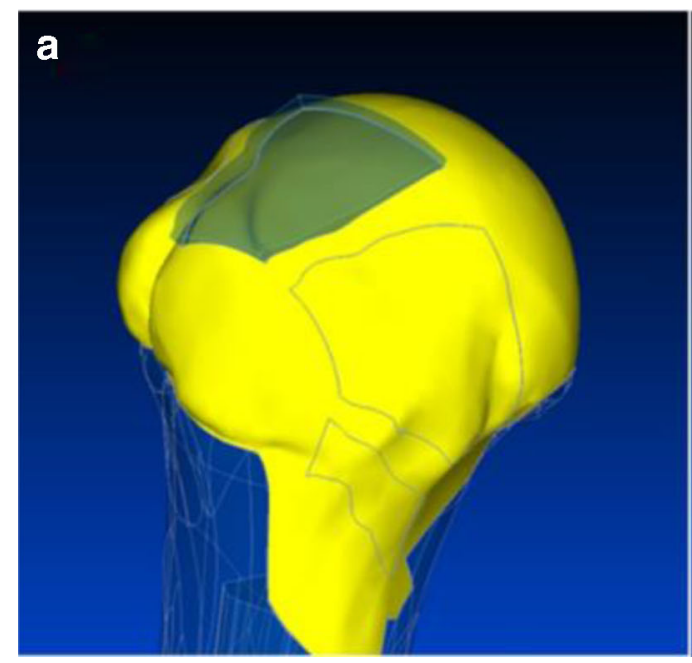

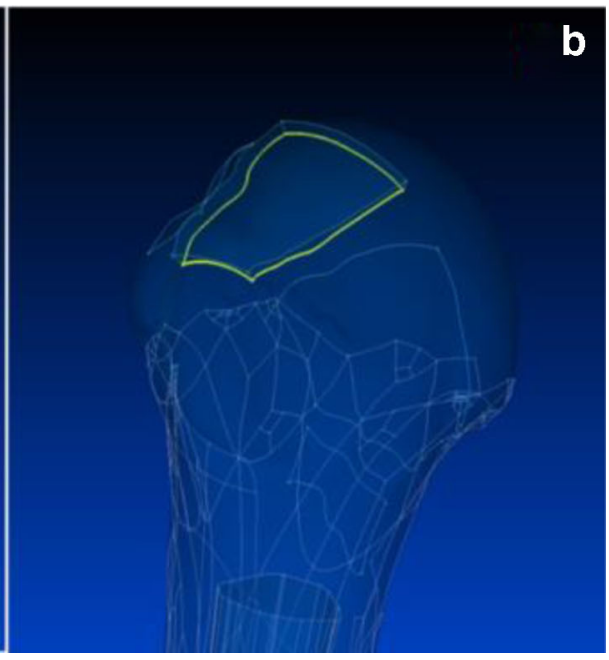


Fig. 13 Mesh Sizing

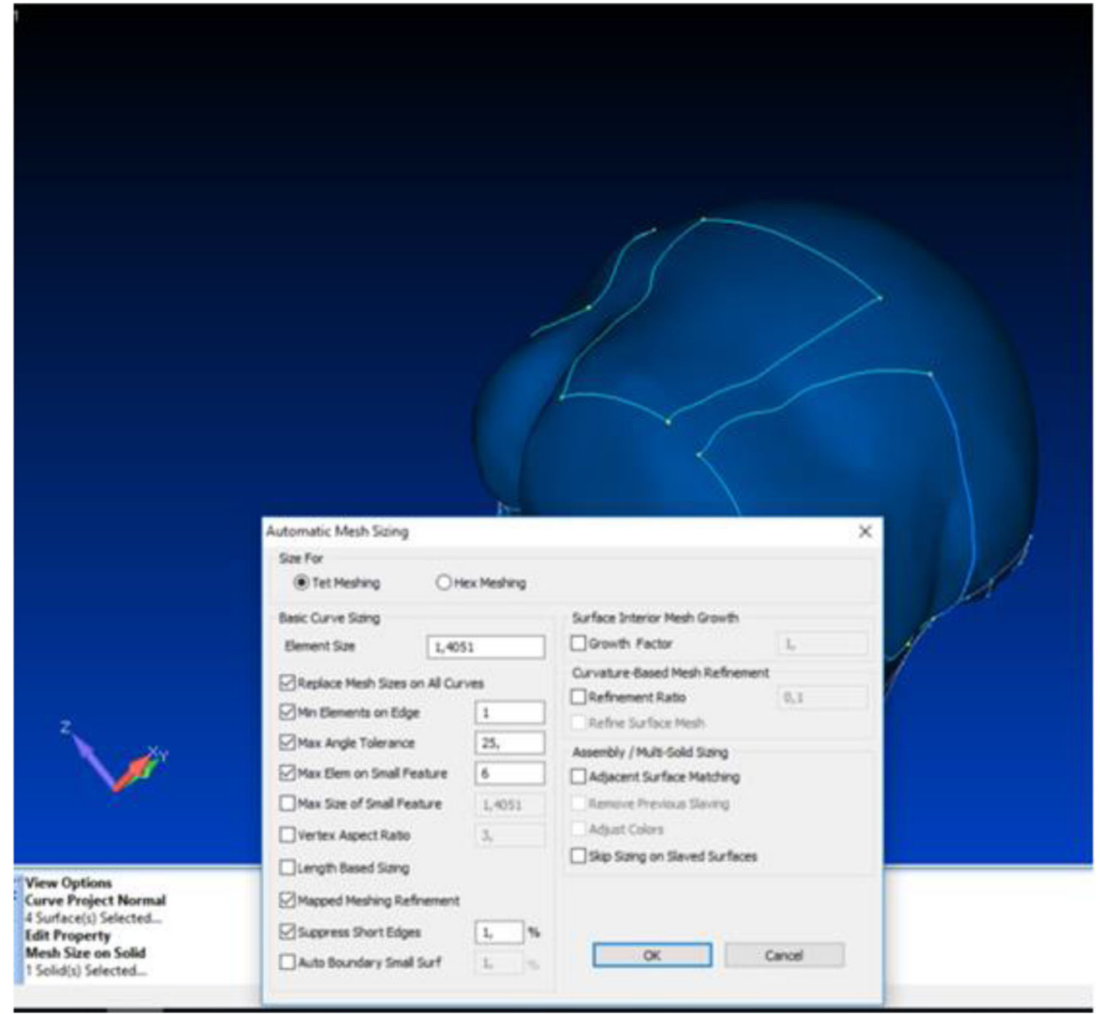

on the cloud of points to build the outer surface of the piece.

- Based on geometrical operations. Geomagic Design X looks for geometrical surfaces in the point cloud (cylinders, flat faces, etc. ...) to reconstruct the piece.

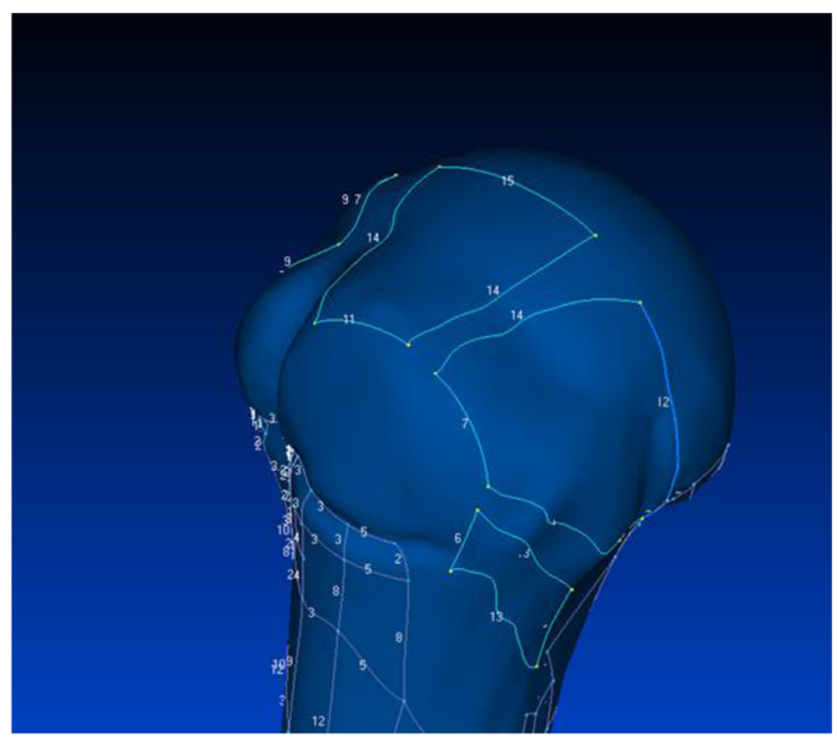

Fig. 14 Number of nodes in each curve
In the case of complicated geometrical pieces, it is recommended to use patch reconstruction. Locating known geometries in a piece as the scapula is complicated so the patches are a suitable solution to obtain the surface of the piece (Fig. 4).

The result of the reconstruction is shown in Fig. 5. The surface of the piece is composed of a series of patches, surfaces of different sizes, which adapt to the

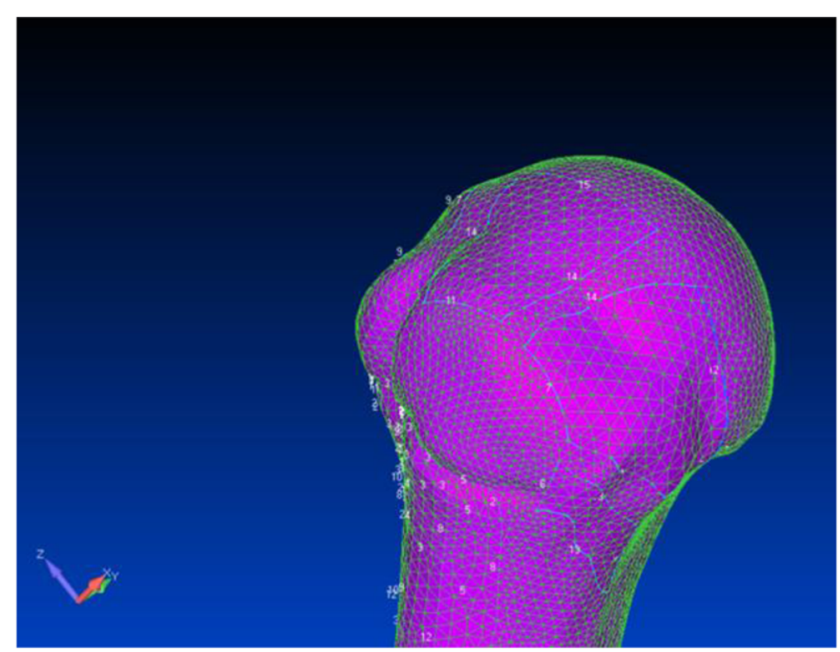

Fig. 15 Obtained mesh 
Fig. 16 Nodes located on the tendon-bone interface surface corresponding to the Humerus

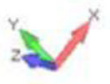

cloud of points, thus creating the outer surface of the piece and, therefore, its volume.

To verify that the interfaces between tendons and bones are perfectly matched, a 3D CAD, NX tool from Siemens has been used. The basis of the meshing process in these areas is the coincidence of the meshes between both surfaces in contact. To do this, a modeling work on the tendons that ends with a Boolean operation has been carried out.

Fig. 17 Nodes located on the tendon-bone interface surface corresponding to the tendon

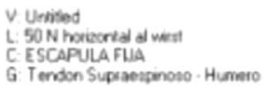

Taking as an example the insertion of the supraspinatus tendon in the head of the humerus, it is possible to explain the process of modeling the tendon in order to achieve a perfect match. Figure 6a shows that the tendon has a thickness greater than the real one, so that between the tendon and the bone there is an interference between both pieces. by means of a Boolean subtraction operation it is possible to subtract the interference to the tendon volume. As shown in

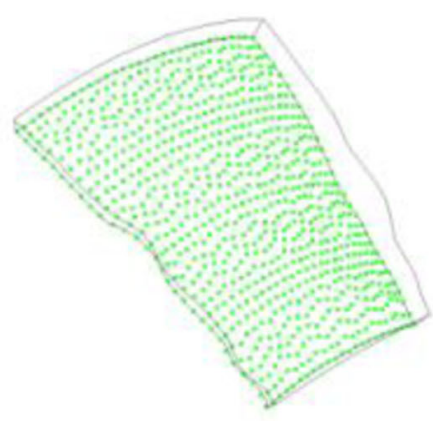


Fig. 18 Nodes fused between the two coinciding surfaces of the tendon and the Humerus

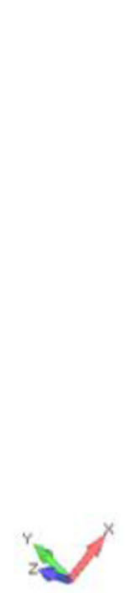

Fig. 6b, it is enough select the two volumes so that Geomagic Design X automatically will calculate the volume of the intersection. The volume of the intersection, the interference between both bodies, is observed in Fig. 6c. subtracting the volume from the tendon, a modeled tendon is obtained with the support surface between the head of the humerus and the coincident tendon between both bodies (Fig. 6d).

This Boolean subtraction operation must be carried out in all the tendons involved in the articulation so that the coincidence between them and the surface in which they are inserted in the bones is perfect. This coincidence is necessary so that during the process of meshing the finite elements a mesh is obtained that shares nodes between the tendons and the bones so that the insertion of the same is modelled correctly.

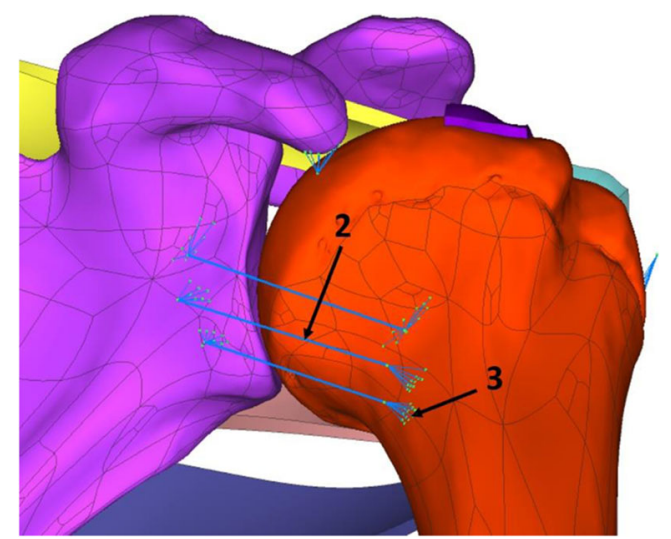

Fig. 19 Rigid element (3) and element 1D type Beam (2) to simulate ligaments of the joint (Table 1)

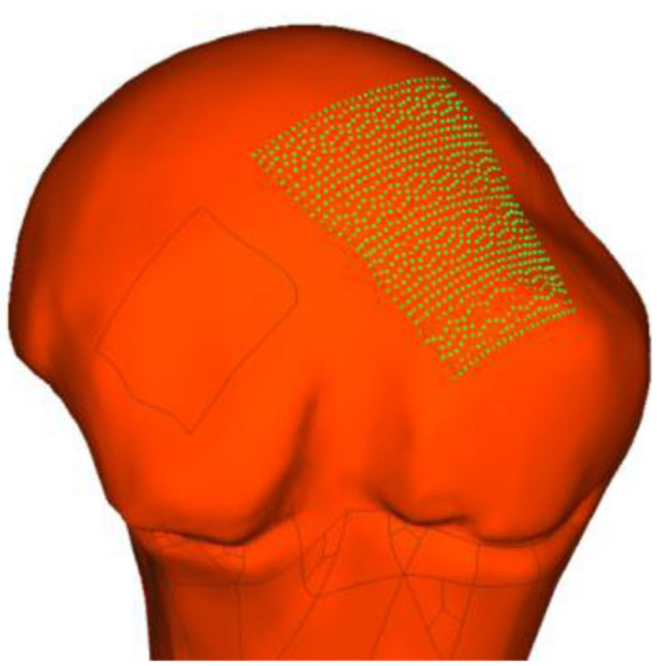

After performing the modelling operations in which the match between tendons and bones is checked, the obtained model is shown in Figs. 7 and 8.

With the solid models of the articulation elements, a file is generated "*.stp" or "*.igs". These files are imported into a FEM software (Siemens FEMAP). As in the case described before, it must be ensured matching surfaces between tendon and bone (Fig. 9). In this way both surfaces will be coincident in geometry (achieved with the Boolean operation in the CAD $3 \mathrm{D}$ software) and also in the contour of them (Fig. 10). Once verified that the geometries between bone and tendon are coincident, the model mesh is generated. Table 1 summarizes the

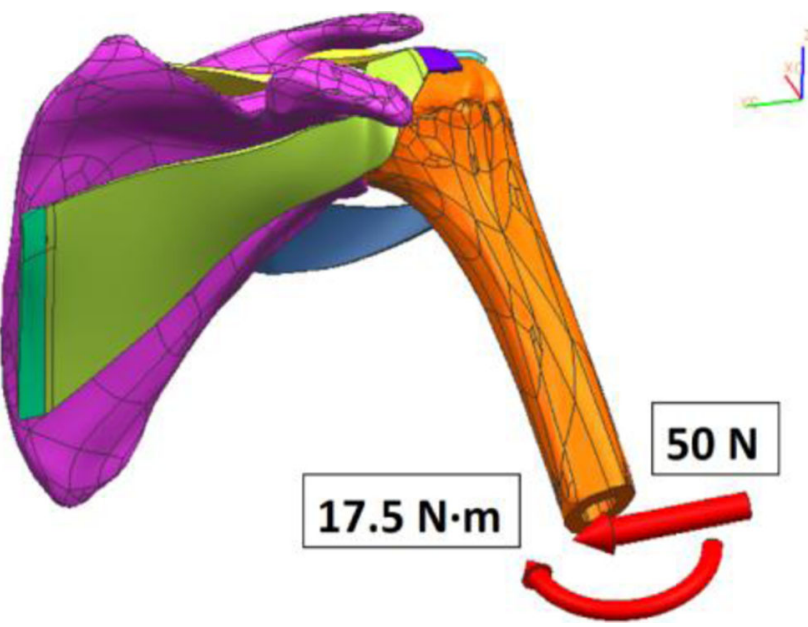

Fig. 20 Load case 1 used in finite element model 
Table 2 Materials used for the model

\begin{tabular}{crll}
\hline & Young's Modulus (MPa) & Poisson & Density $\left(\mathrm{Kg} / \mathrm{m}^{3}\right)$ \\
\hline Bone & 17,200 & 0,41 & 1790 \\
Muscle & 0,3 & 0,43 & 1350 \\
Tendon & 2000 & 0,43 & 1350 \\
Cartilage & 8 & 0,49 & 1150 \\
Ligament & 300 & 0,43 & 1350 \\
\hline
\end{tabular}

elements used to model the parts which the human shoulder joint consists of.

This projection of the contour of the tendons on the surface of the bones is achieved in Siemens FEMAP by projecting the curves that make up the contour of the tendon on the insertion surface in the bone (Fig. 11). Then select the surfaces on which the curves are going to be projected, in our case shown in yellow in the Fig. 12a. Finally, the curves wish to project on the surface are selected, in our case, the curves that define the contour of the tendon (Fig. 12b).

The projection is produced according to the normal to the surface at each point, it is also possible to project the curves according to a defined vector. The result of the projection is a partition on the surface (Fig. 13).

Once this operation of projection of curves on the surfaces is done, the solids are obtained in situation of starting the process of generating the finite element mesh to proceed to the calculation of the different load cases. The meshing process is carried out using the types of elements for each body that intervenes in the joint, see Table 1. To generate the mesh must follow the following steps:

1. Generation of the physical properties of bodies. Consisting of defining the physical characteristics of the finite elements used:

a) Characteristics of the material that defines the element, bone, tendon, muscle, etc.

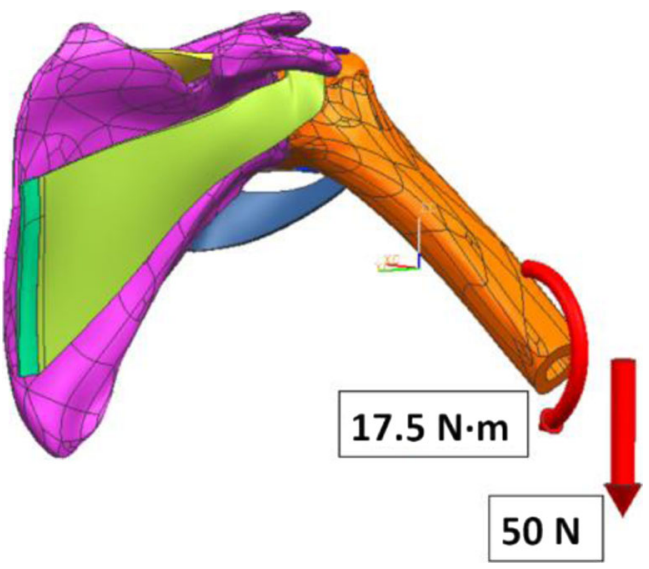

Fig. 21 Load case 2 used in finite element model

b) Geometrical characteristics of the element, thickness (in the case of using elements $2 \mathrm{~d}$ ), section (in the case of using elements $1 \mathrm{~d}$, elastic constant (in the case of using spring elements) ....

2. A different physical property is assigned to each body involved in the articulation, in this way it is necessary to create as many physical properties as bodies participate in the model.

Once the physical properties of the bodies involved in the articulation have been defined, meshing is carried out. The first step is to define the size of the element to be used, the smaller element is the more accurate the analysis, it is worth mentioning that the calculation process by means of the finite elements method obtains results in the defined nodes, the rest of the model, the spaces without nodes, are calculated by extrapolation between nodes depending on the type of element, and its characteristics, that unites them with each other. However, the smaller the selected element size, the greater the time and resources needed for the calculation, that is why the number of nodes to be used should be minimized, keeping the minimum size necessary to

Table 3 Load cases considered for FEA

\begin{tabular}{clc}
\hline $\begin{array}{l}\text { Load } \\
\text { Case }\end{array}$ & Load Conditions & Constrain Conditions \\
\hline 1 & Gravity applied negative $\mathrm{Z}$ axis $\left(9.841 \mathrm{~m} / \mathrm{s}^{2}\right)$ & $\begin{array}{c}\text { Fixed scapula on areas shown } \\
\text { in Fig. 20 }\end{array}$ \\
2 & $\begin{array}{c}\text { Gravity applied along negative } \mathrm{Z} \text { axis }\left(9.841 \mathrm{~m} / \mathrm{s}^{2}\right)+50 \mathrm{~N} \text { applied } \\
\text { along positive } \mathrm{Y} \text { axis. } \\
\text { Gravity applied along negative } \mathrm{Z} \text { axis }\left(9.841 \mathrm{~m} / \mathrm{s}^{2}\right)+50 \mathrm{~N} \text { applied } \\
\text { along negative } \mathrm{Z} \text { axis. }\end{array}$ & $\begin{array}{c}\text { Fixed scapula on areas shown } \\
\text { in Fig. 21 } \\
\text { Fixed scapula on areas shown } \\
\text { in Fig. 22 }\end{array}$ \\
\hline
\end{tabular}



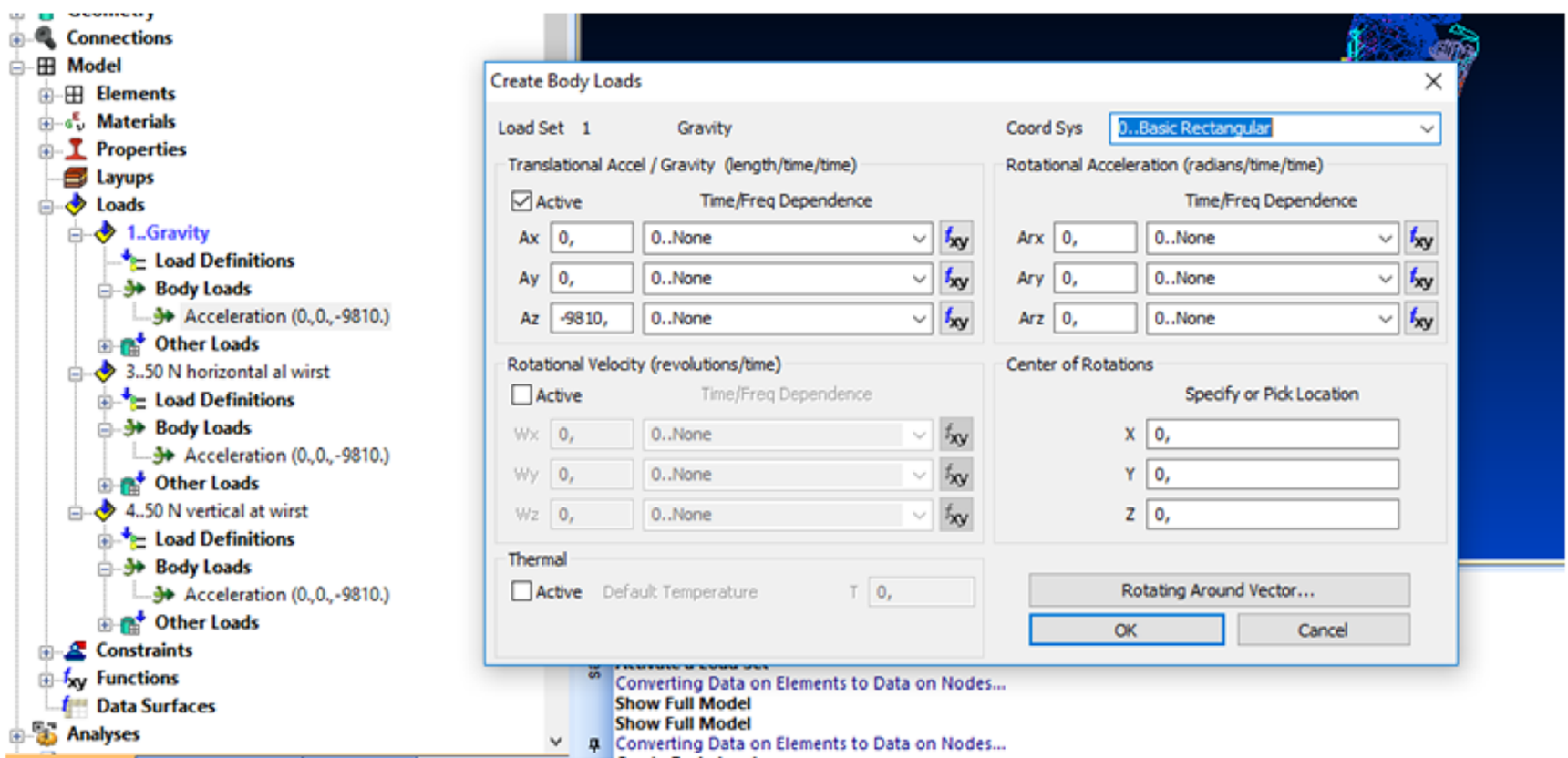

Fig. 22 Effect of gravity applied to finite element model

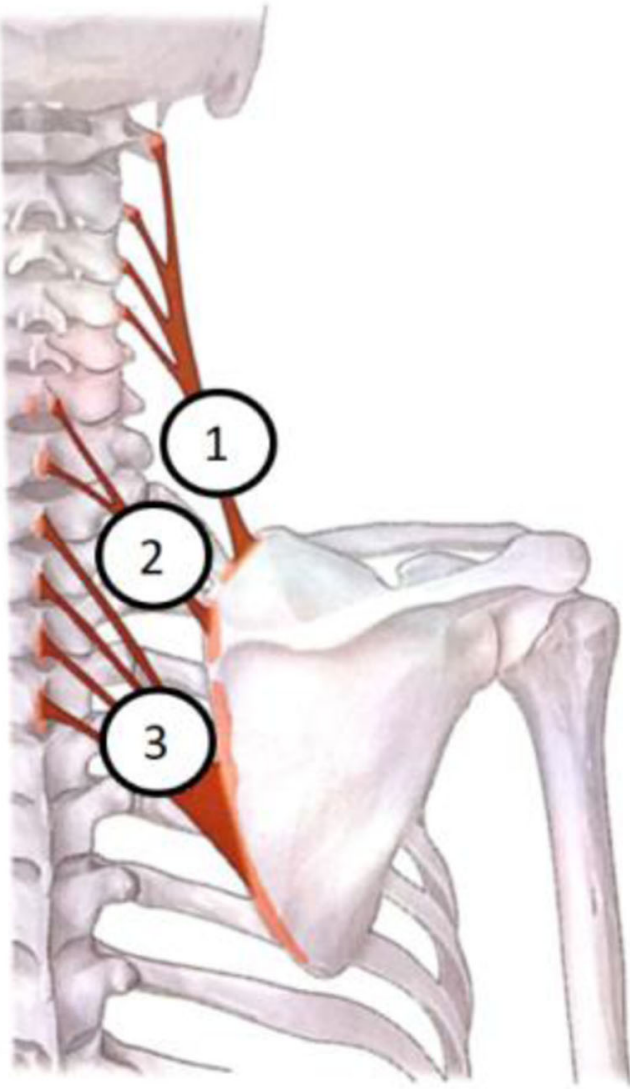

Fig. 23 Muscles attached to the scapula fixed reproduce with precision the geometry of a discrete mode and a finite number of nodes. To define the element size, select the desired volume and assign it a size. Siemens FEMAP is responsible for locating the nodes in that volume in the optimal way (Fig. 13).

Once the number of nodes that make up the piece have been defined, the mesh of elements is generated. Figure 14 shows the number of nodes that have been created in each curve that defines the body, Siemens FEMAP is responsible for generating the nodes inside the surfaces by interpolation and triangulation of them. After the meshing process of the body the result obtained is a mesh, composed of nodes and elements, which define the geometry of the body (Fig. 15). The process must be repeated in all the bodies that make up the joint.

Once the mesh is generated separately from the muscles, tendons, bones and ligaments, the next step is the fusion of the matching nodes to generate the complete model. As an example, the case of the interface between bone and tendon is of special importance (Figs. 16 and 17). In fact, with this fusion it is possible to transfer the forces between both elements through matching nodes between both surfaces (Fig. 18). This avoids modelling contacts between them.

To improve the response of the FEM proposed, the ligaments that form part of the human shoulder joint have been considered. These ligaments are: 
Fig. 24 Areas of the scapula without displacement, in red

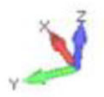

1. Superior glenohumeral ligament: medially inserted into the top of the glenoid labrum and neck of the scapula and extends to the base of the coracoid process.

2. Middle glenohumeral ligament: it is inserted medially in the glenoid rim and in the neighbouring part of the neck of the scapula, to end in the lower part of the minor tubercle, along the insertion of the subscapularis tendon.

3. Lower glenohumeral ligament: reinforces the anteroinferior part of the joint capsule. It is inserted into the glenoid rim and reaches to the lower part of the lower tubercle of the humerus.

4. Coracohumeral ligament: thick fibrous lamina, which connects the humeral head with the coracoid process of

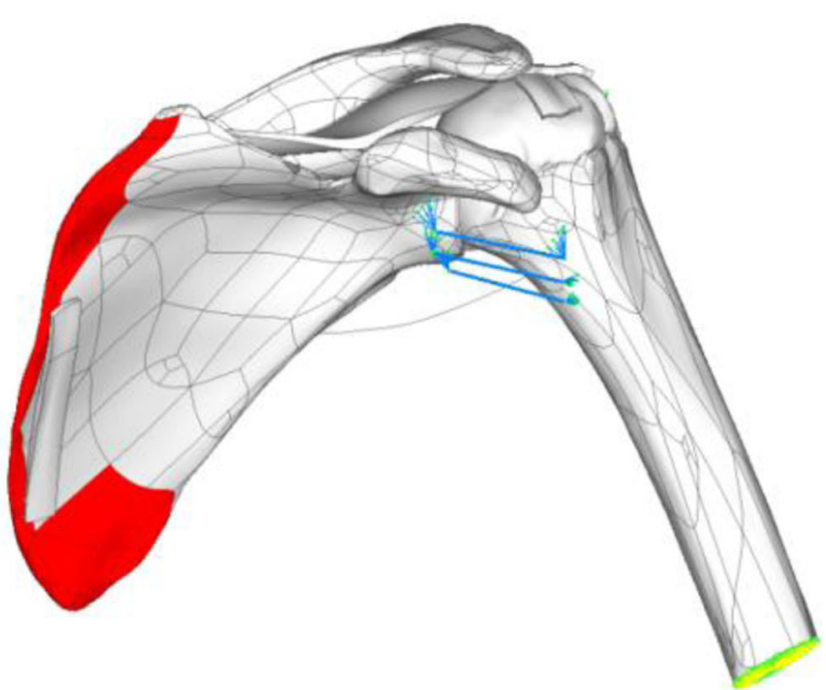

the scapula, which generates tension and stability when the arm is on the side of the body. It merges with the capsule of the shoulder joint.

Similarly, it has been included in the model the glenohumeral capsule wrapping the joint formed between the humerus and the scapula.

For the ligaments, 1D beam elements have been used, where the section of the element is that of the real ligament. The use of rigid elements to simulate the insertion of the ligament in the bone allows to eliminate the concentration of tensions that would occur if the insertion were made in a single node (Fig. 19).
Fig. 25 Areas of the scapula without displacement, in red

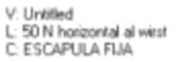

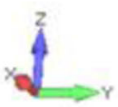

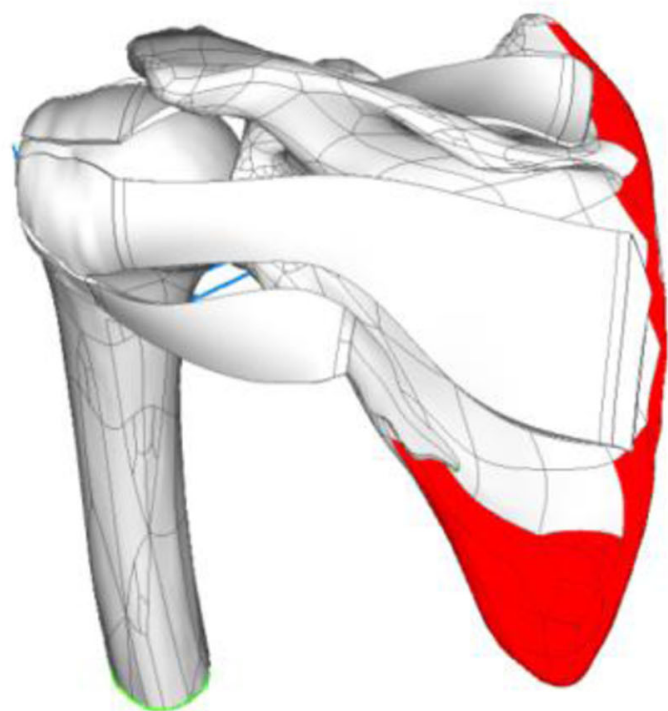




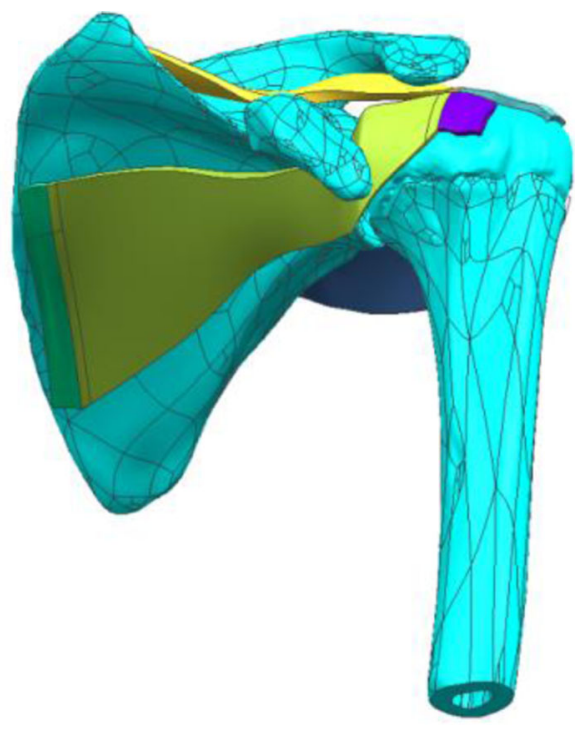

Fig. 26 CAD model from the cloud of points (frontal view)

The materials used for the model are summarized in Table 2. In this study three load cases have been considered (Table 3).

For the proposed finite element model has considered the effect of gravity. This has been applied as shown in Fig. 22.

For the boundary conditions, the scapula was considered fixed in the area where the muscles of the scapula would be inserted. These muscles are (Fig. 23):

1. Levator scapula muscle.

2. Rhomboid minor muscle.

3. Rhomboid major muscle.

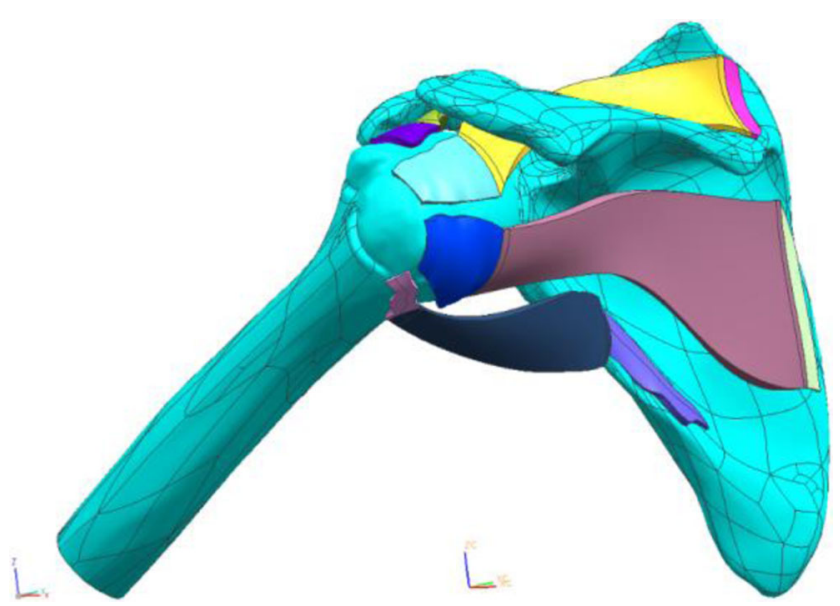

Fig. 27 CAD model from the cloud of points (rear view)

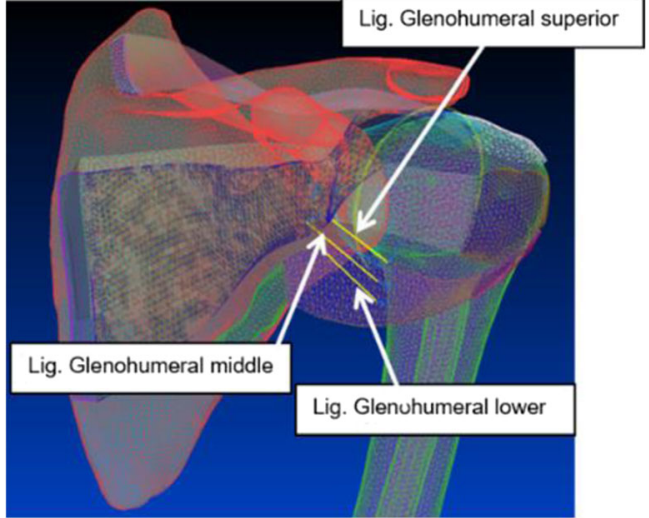

Fig. 28 Detail of glenohumeral ligaments modeled as beam elements, in yellow

Figs. 24 and 25 show the areas of the scapula without displacements applied to the developed FEM. Fig. 24 shows the scapula in the front view and Fig. 25 in the rear view.

\section{Results and discussion}

This section shows the results of the meshed geometric model and the finite element analysis implemented in this study.

\section{Computer Aided Design (CAD) model}

Figures 26 and 27 show the CAD model obtained from the clouds of points.

From this CAD model, the corresponding mesh has been generated for the FEA, in which all the elements mentioned in Table 1 are included.

As shown in Fig. 28, the generated mesh is suitable for carrying out the finite element analysis. The combination of $3 \mathrm{D}$ elements and $1 \mathrm{D}$ elements used meets the objectives of the study. In fact, the main objective is the adequacy of the mesh so that the finite element method simulation reproduces the behaviour of the ligaments in the model. The stresses that appear in bones, muscles, cartilage area and insertions of the ligaments are consistent with the expected result.

\section{Finite Element Analysis (FEA)}

The results shown in Figs. 29, 30 and 31 represent the stresses and displacements in the articulation under study by colour coding. The colours vary from the maximum value represented in red to the minimum value represented in blue. 

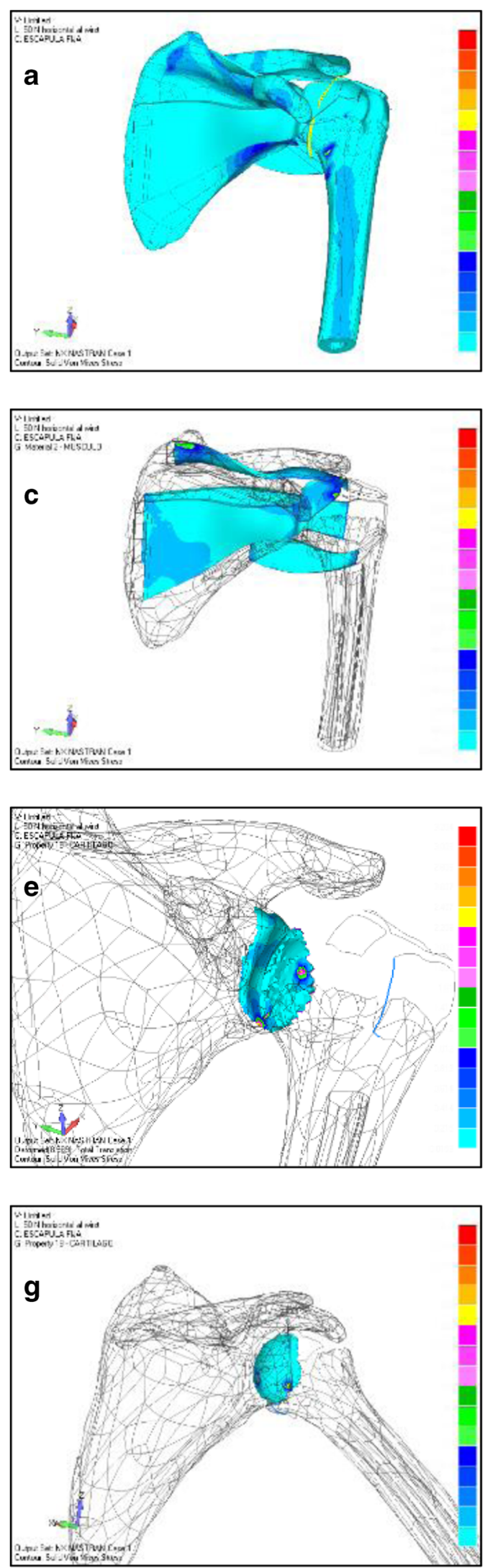

Fig. 29 Load case 1, FEM results: a) Stresses in complete model; b) Maximum stresses in bones; c) Stresses in muscles; d) Stresses in tendons; e) Stresses in cartilage zone, scapula-Humerus; f) Stresses in
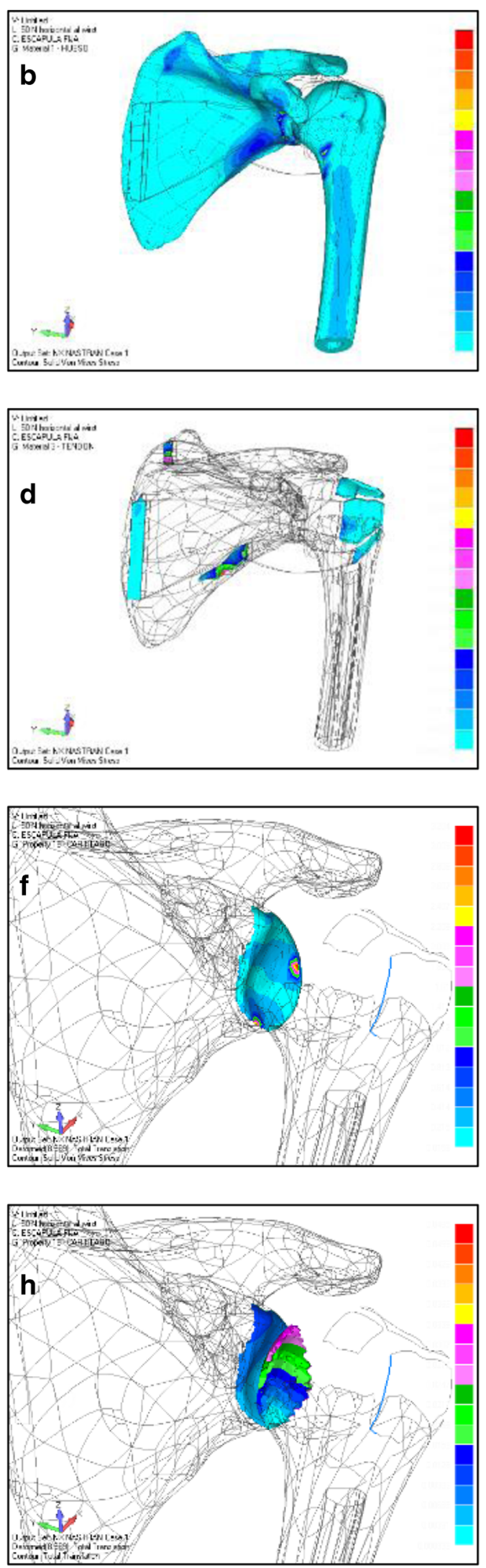

cartilage, only Humerus; g) Stresses in cartilage zone, only scapula; h) Displacements in cartilage zone 

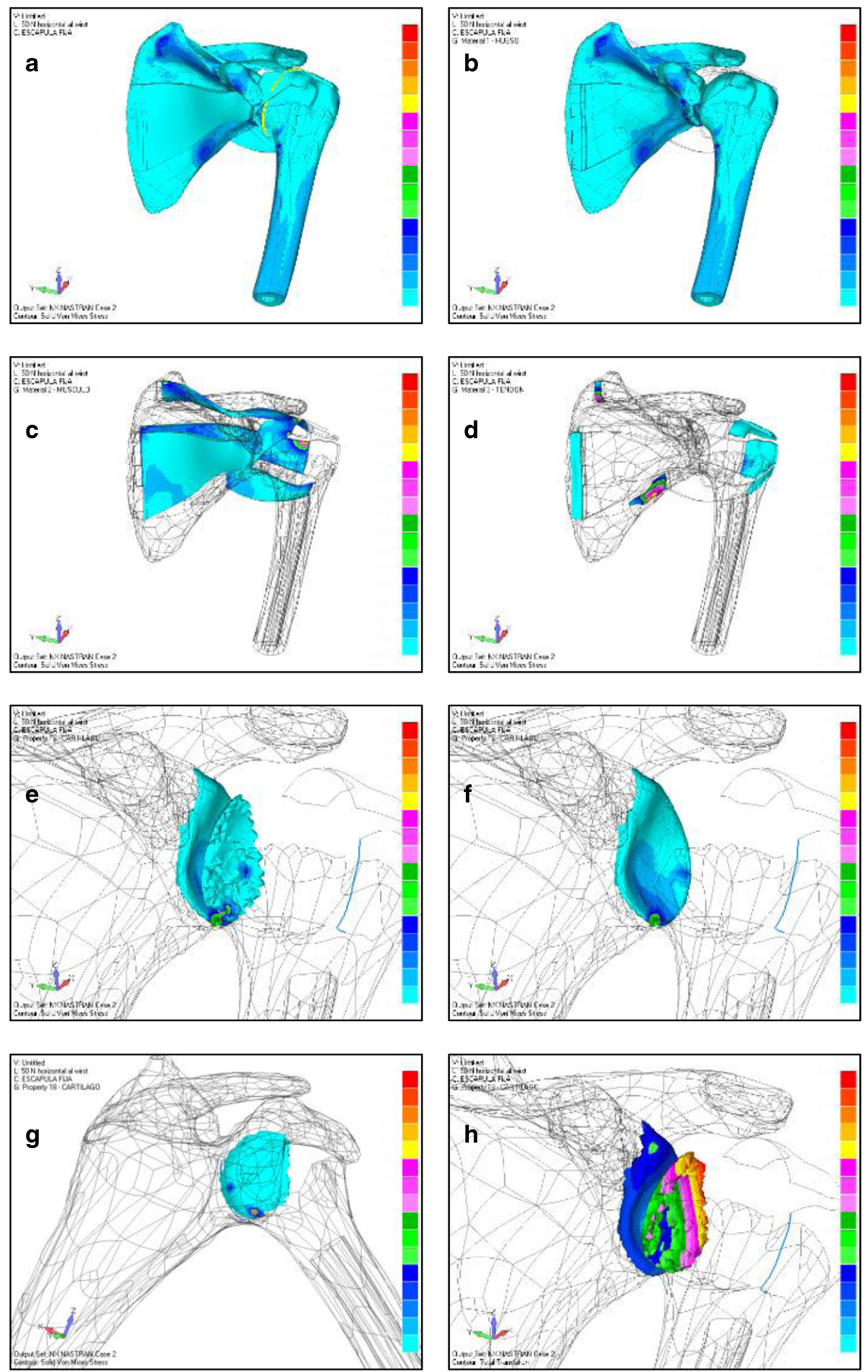

Fig. 30 Load case 2, FEM results: a) Stresses in complete model; b) Maximum stresses in bones; c) Stresses in muscles; d) Stresses in cartilage, only Humerus; g) Stresses in cartilage zone, only scapula; h) Displacements in cartilage zone 

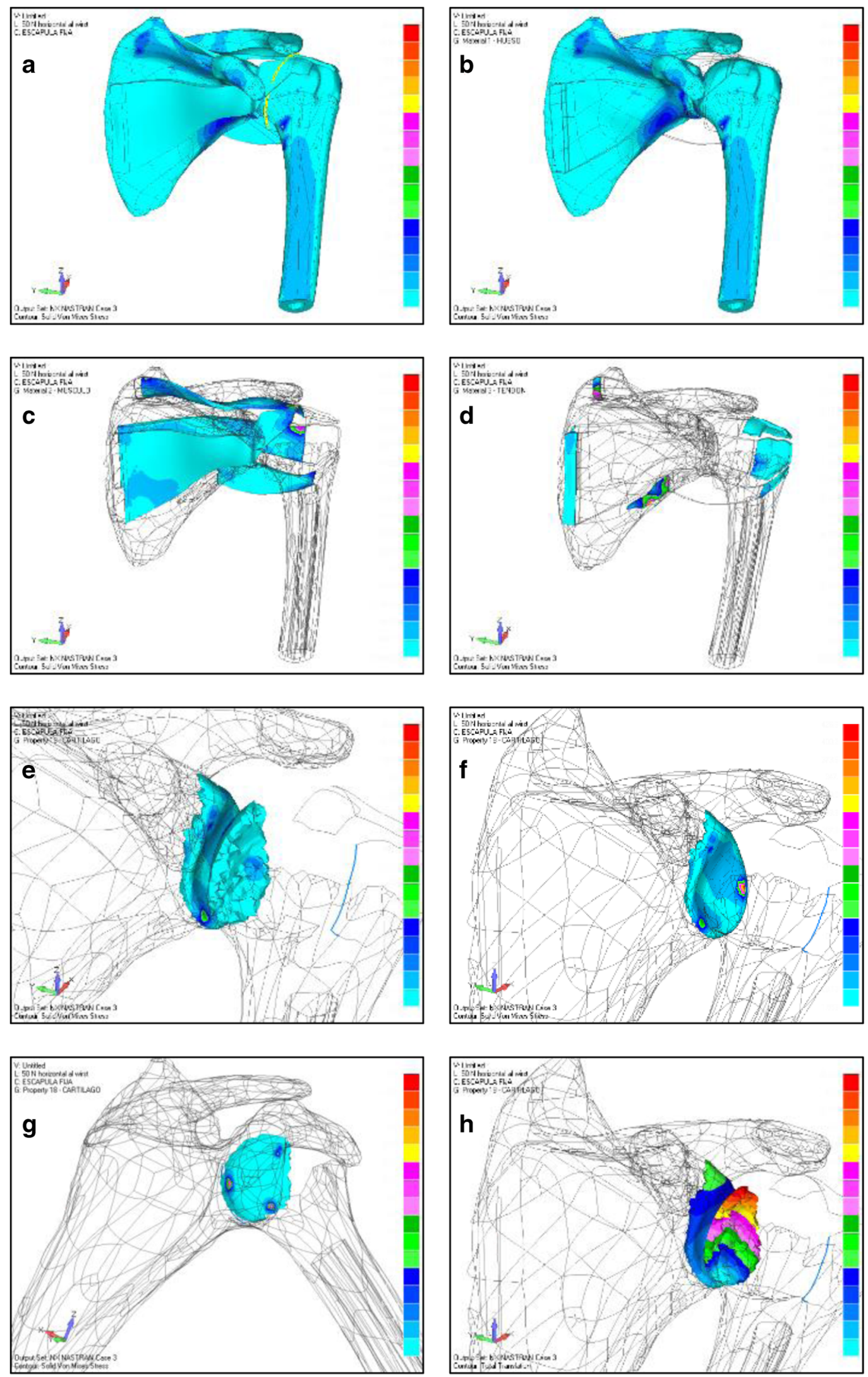

Fig. 31 Load case 3, FEM results: a) Stresses in complete model; b) Maximum stresses in bones; c) Stresses in muscles; d) Stresses in tendons; e) Stresses in cartilage zone, scapula-Humerus; f) Stresses in

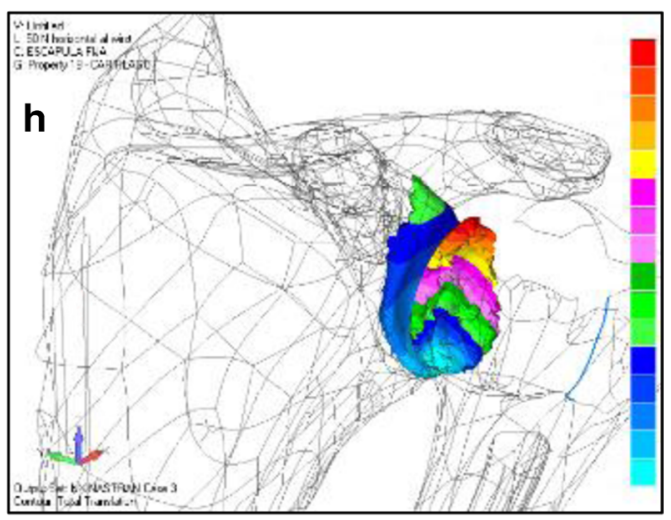

cartilage, only Humerus; g) Stresses in cartilage zone, only scapula; h) Displacements in cartilage zone 


\section{Stresses in bones}

As shown in the figures of the three load cases studied (Figs. 29, 30 and 31, section a to b), the maximum stresses in bones are in the insertion areas of the ligaments. This makes physical sense since ligaments are responsible for keeping the joint in position. Therefore, the ligaments transfer the necessary efforts to the area of insertion of the ligaments in the bones, causing these points to be the most stressed.

\section{Stresses in muscles}

Due to the static positions of the joint under study, the muscles do not support high stresses (Figs. 29, 30 and 31, section c). According to the stresses distribution, the maximum values are in the zones of tendons (Figs. 29, 30 and 31, section d).

This situation has great physical sense since tendons are responsible for transmitting efforts of muscles to the bone structure while maintaining the position of the joint.

\section{Stresses in cartilage}

In the cartilage zone (Figs. 29, 30 and 31, section e, f and g) a uniform and low value stresses distribution is observed. However, points with high stress concentration does not have a great physical sense, they are due to the alignment of the gap element. As the gap element, behaves like a compression spring, the stresses that appear in it are proportional to the displacement that occurs in this kind of element (Figs. 29, 30 and 31 , section h). Observing the images of the displacements that occur around the cartilage (Figs. 29, 30 and 31 , section $\mathrm{h}$ ) it is verified how they adjust to the expected, producing the displacement of the head of the Humerus. This displacement moves the head away from the Humerus of the scapula, which in comparison remains with very low values. However, if the gap element alignment, in relation to the displacement that occurs, is not correct, high stresses may appear that do not correspond to reality. Despite this, it is observed how the stresses distribution in the cartilage area maintains coherence with reality since the maximum stresses appear in the lower half of the cartilage. In this area in which the Humerus-scapula contact is greater, the compression tensions are greater.

\section{Conclusions}

This study validates the FEM developed presenting a first qualitative study of the performances of the joint of the human shoulder. The proposed analysis meets the stated objective: a) Obtaining an improved mesh of the shoulder joint to analyse real situations with application in the field of sports medicine, work, etc. For this reason, the follow improvements have been carried out:

- Meshing of the joint, bone, tendons and muscles as 3D elements.

- Improved mesh in the insertion of tendon-bone.

- Congruence between the matching meshes of the tendon insertion area with the bone.

- Including ligaments as 1D elements, node to node.

b) Obtaining congruent and logical results of the state of load of the articulation in the three proposed cases.

- Simplifying the modelling of muscles. It is of great interest model muscles simplified to $1 \mathrm{D}$ elements. It makes possible to establish different positions in the articulation without having to mesh again each one of the positions that can be studied.

c) This improvement must consider:

- Young's modulus of muscle is not linear. This depends on the load to which the muscle is subjected and its length

- Muscle geometry (cross-section) varies depending on the position in which it is located.

- Modifying the model used to simulate the ligaments considering that the strain-stress curve of them is not linear along its entire length. This involves using 1D elements to adapt its Young's modulus to the length having the ligament at each time.

- Improving simulation at the contact zone cartilage-bones of the joint. It must be investigated about the possible improvement of the alignment of the gap elements.

The final objective of the study is to obtain an FEM to implement different positions of the same joint without modifying the initial mesh. This requires use $1 \mathrm{D}$ elements for the anatomical elements that deform, ligaments and muscles, and $3 \mathrm{D}$ elements for bones and tendons involved in the joint. The FEM model developed demonstrate that the results are consistent with the states of applied loads in the human joint under real work conditions. In fact, the maximum stresses in bones are in the insertion areas of the ligaments. Due to the static positions of the joint under study, the muscles do not support high stresses. According to the stresses distribution, the maximum values of these one are in the zones of tendons. From the result analysis, it is observed how the stresses distribution in the cartilage area maintains coherence with reality since the maximum stresses appear in the lower half of the cartilage. In this area in which the Humerus-scapula contact is greater, the compression tensions are greater. 


\section{Compliance with ethical standards}

Conflict of interest Manuel Islán Marcos declares that he has no conflict of interest. Emilio Lechosa Urquijo declares that he has no conflict of interest. Fernando Blaya Haro declares that he has no conflict of interest. Roberto D'Amato declares that he has no conflict of interest. Enrique Soriano Heras declares that he has no conflict of interest. Juan Antonio Juanes declares that he has no conflict of interest.

Ethical approval This article does not contain any studies with human participants performed by any of the authors. This article does not contain any studies with animals performed by any of the authors.

\section{References}

1. Brunelli, M. P., and Gill, T. J., Fractures and tendon injuries of the athletic shoulder. Orthop. Clin. North Am. 33(3):497-508, 2002.

2. Islan, M., Carvajal, J., Pedro, P. S., D'Amato, R., Juanes, J. A., and Soriano, E., Linear approximation of the behavior of the rotator cuff under fatigue conditions. Violinist case study, in Proceedings of the 5th International Conference on Technological Ecosystems for Enhancing Multiculturality - TEEM 2017, pp. 1-8, 2017.

3. M. Islan, F. Blaya, P. San Pedro, R. D'Amato, E. Lechosa Urquijo, and J. A. Juanes, "Analysis and fem simulation methodology of dynamic behavior of human rotator cuff in repetitive routines: Musician case study," J. Med. Syst., vol. 42, no. 3, 55, 2018.

4. CDC - NIOSH Publications and Products, Cumulative trauma disorders in the workplace (95-119), DHHS Publ. Number 95-119, 1995.

5. International Labour Office - Geneva, LIST OF OCCUPATIONAL DISEASES (revised 2010) Identification and recognition of occupational diseases: Criteria for incorporating diseases in the ILO list of occupational diseases. Geneva: INTERNATIONAL LABOUR OFFICE GENEVA, 2010.

6. Ruggiero, A., Affatato, S., Merola, M., and De, M. C., FEM analysis of metal on UHMWPE total hip prosthesis during normal walking cycle, in Proceedings of the XXIII Conference of the Italian Association of Theoretical and Applied Mechanics (AIMETA 2017), pp. 4-7, 2017.

7. Ruggiero, A., Gòmez, E., and D'Amato, R., Approximate analytical model for the squeeze-film lubrication of the human ankle joint with synovial fluid filtrated by articular cartilage. Tribol. Lett. 41(2): 337-343, 2011.

8. Ruggiero, A., Gómez, E., and D'Amato, R., Approximate closedform solution of the synovial fluid film force in the human ankle joint with non-Newtonian lubricant. Tribology International 57: 156-161, 2013.

9. D'Amato, R., Calvo, R., and Gómez, E., Sensitivity study of the morphometric fitting on the pressure field inside ankle joints. Case Stud. Mech. Syst. Signal Process. 1:8-14, 2015.

10. Luo, Z. P., Hsu, H. C., Grabowski, J. J., Morrey, B. F., and An, K. $\mathrm{N}$., Mechanical environment associated with rotator cuff tears. J. shoulder Elb. Surg. 7(6):616-620, 1998.

11. Seki, N. et al., Mechanical environment of the supraspinatus tendon: Three-dimensional finite element model analysis. J. Orthop. Sci. 13(4):348-353, 2008.

12. Zheng, M., Zou, Z., Bartolo, P. J. D. S., Peach, C., and Ren, L., Finite element models of the human shoulder complex: a review of their clinical implications and modelling techniques, International Journal for Numerical Methods in Biomedical Engineering, vol. 33, no. 2. Wiley-Blackwell, 2017.

Publisher's Note Springer Nature remains neutral with regard to jurisdictional claims in published maps and institutional affiliations. 